\title{
Cellular neurothekeoma of child, a new case
}

\section{Fatima Zahra Elfatoiki ${ }^{1}$, Fayrouz Cherkaoui ${ }^{1}$, Hayat Dahbi Skali', Farida Marnissi², Fouzia Hali', Soumia Chiheb'}

${ }^{1}$ Department of Dermatology, Ibn Rochd UHC, 1 quartier des hopitaux 20000, Casablanca, Morocco, ${ }^{2}$ Department of Anatomo-Pathology, Ibn Rochd UHC, 1 quartier des hopitaux 20000, Casablanca, Morocco

Corresponding author: Dr. Fatima Zahra Elfatoiki, E-mail: Fatiza59@hotmail.fr

\begin{abstract}
Cellular neurothecoma is a benign skin tumor. We report a new observation in a child. A 12-year-old patient presented in our department for an asymptomatic nodular lesion of the left sternocleidomastoid region that had been evolving for two years. The skin biopsy showed a cellular neurothecoma. Surgical excision was performed.

Cellular neurothecoma is an entity that deserves to be known in order to distinguish it from the main differential diagnosis in the child which is the Spitz nevus.
\end{abstract}

Key words: Neurothecoma; Skin tumor; Benign 


\title{
Neurothécome cellulaire de l'enfant, une nouvelle observation
}

\section{Fatima Zahra Elfatoiki ${ }^{1}$, Fayrouz Cherkaoui ${ }^{1}$, Hayat Dahbi Skali', Farida Marnissi², Fouzia Hali ${ }^{1}$, Soumia Chiheb ${ }^{1}$}

${ }^{1}$ Department of Dermatology, Ibn Rochd UHC, 1 quartier des hopitaux 20000, Casablanca, Morocco, ${ }^{2}$ Department of Anatomo-Pathology, Ibn Rochd UHC, 1 quartier des hopitaux 20000, Casablanca, Morocco

Corresponding author: Dr. Fatima Zahra Elfatoiki, E-mail: Fatiza59@hotmail.fr

\begin{abstract}
RÉSUMÉ
Le neurothécome cellulaire est une tumeur cutanée bénigne. Nous rapportons une nouvelle observation chez l'enfant. Patiente de 12 ans s'est présenté dans notre département pour une lésion nodulaire asymptomatique de la région sterno-cléido-mastoïdienne gauche évoluant depuis deux ans. La biopsie cutanée était en faveur d'un neurothécome cellulaire. Une exérèse chirurgicale était pratiquée. Le neurothécome cellulaire est une entité qui mérite d'être connue afin de la distinguer du principal diagnostic différentiel chez l'enfant qui reste le nævus de spitz.
\end{abstract}

Mots clés: Neurothécome; Tumeur cutanée; Bénigne

\section{INTRODUCTION}

Le neurothécome cellulaire est une tumeur cutanée bénigne rare de l'adulte jeune, exceptionnelle chez l'enfant. Son diagnostic est histologique. C'est une entité qui mérite d'être connue par les pédiatres et les dermatologues afin de la distinguer de certaines tumeurs malignes notamment fibro-histiocytaires, sarcomateuses et mélanocytaires. Nous rapportons une nouvelle observation de neurothécome cellulaire chez une patiente de 12 ans.

\section{OBSERVATION}

Patiente de 12 ans sans antécédents notables consultait pour une tumeur de la région sternocléido-mastoïdienne gauche évoluant depuis deux ans. L'examen clinique objectivait une lésion nodulaire unique de couleur chair d'environ $0,5 \mathrm{~cm}$ de diamètre, indolore, de consistance ferme, non adhérente au plan profond et non fistulisée (Fig. 1). Il n'y avait aucune autre lésion associée. Les muqueuses étaient normales et les aires ganglionnaires étaient libres. L'examen histologique d'un fragment biopsique avait montré une prolifération cellulaire fusiforme et globuleuse avec des lobules myxoides séparés par des septas collagéniques avec des noyaux augmentés de taille (Figs. 2a and 2b). Létude immuno-histochimique révélait un immunomarquage négatif pour la CKAEl/ AE3, protéine S100, desmine, caldesmone, CD68 et HMB45. Le diagnostic de neurothécome cellulaire était alors retenu. Une exérèse chirurgicale était pratiquée.

\section{DISCUSSION}

Le neurothécome cellulaire est une tumeur bénigne de l'adulte jeune plus fréquente chez la femme. Elle se localise préférentiellement au niveau de l'extrémité céphalique, le tronc et la racine des membres [1].

Elle se présente cliniquement sous la forme d'un nodule généralement unique, d'évolution lente, indolore, ferme à la palpation, de couleur chair, habituellement inférieure à $1 \mathrm{~cm}$, sans ulcération superficielle [2].

Sur le plan histologique, le neurothécome cellulaire a l'aspect d'une tumeur lobulée ou micronodulaire, constituée de nodules ou de courts faisceaux. Les

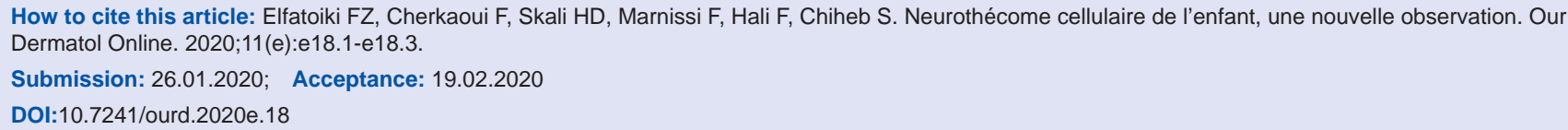




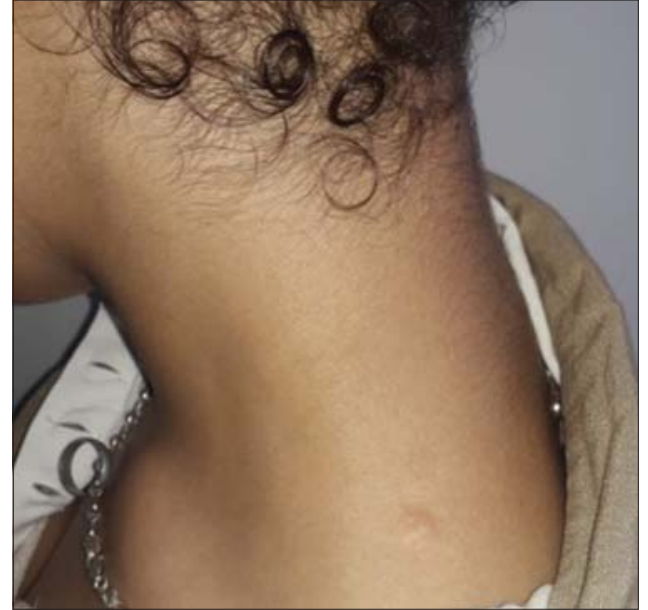

Figure: Lésion nodulaire de couleur chair de la région sterno-cléidomastoïdienne gauche.

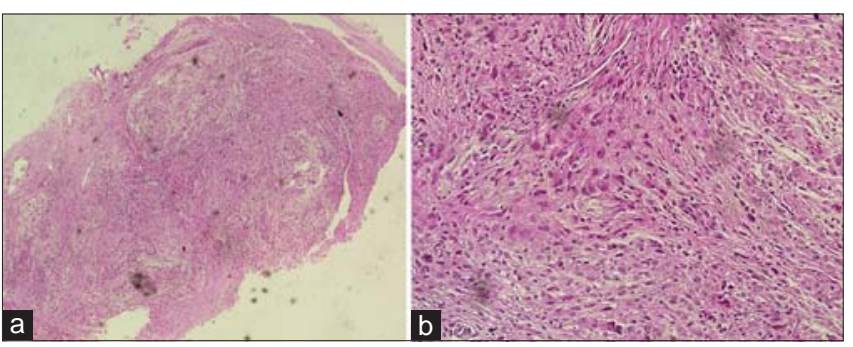

Figure 2: $(a$ and $b)$ Fusiform and globular cell proliferation with myxoid lobules.

cellules tumorales sont fusiformes ou épithélioïdes. Le stroma est collagénique et peut comporter des foyers myxoïdes. Des atypies cyto- nucléaires, une activité mitotique élevée et/ou une infiltration du tissu sous-cutané peuvent être observées de façon occasionnelle [3].

Il est judicieux de distinguer cette entité du neurothécome myxoide d'origine nerveuse caractérisé par une hypocellularité et un abondant stroma alcianophile [3].

Devant l'aspect cellulaire fusiformes et épithélioïdes, le neurothécome cellulaire peut poser un problème de diagnostic différentiel avec des tumeurs fibreuses, histiocytaires, mélanocytaires, musculaires et nerveuses notamment malignes. Le diagnostic de confirmation est posé à l'étude immuno-histochimique [3]

En immuno-histo-chimie, la présence ou l'absence de marquage cellulaire permet de différencier les formes myxoïdes des formes cellulaires du neurothécome et d'assurer le diagnostic différentiel avec les autres tumeurs fibro- histiocytaires, sarcomateuses et mélanocytaires [3].
En pratique, La morphologie de la tumeur et la négativité de la protéine S-100 suffisent à poser le diagnostic. Cette négativité permet d'éliminer le principal diagnostic différentiel histologique du neurothécome cellulaire qui est la tumeur mélanocytaire et en particulier le nævus de Spitz [4].

L'évolution du neurothécome cellulaire est lente. La tumeur peut évoluer pendant plusieurs mois à plusieurs années avant son diagnostic. La prise en charge du neurothécome cellulaire se base sur l'exérèse chirurgicale complète assurée par le contrôle histologique des marges. Après exérèse, l'évolution est favorable. Le neurothécome cellulaire ne récidive que rarement, en particulier en cas d'exérèse incomplète [5].

\section{Consent}

The examination of the patient was conducted according to the Declaration of Helsinki principles.

The authors certify that they have obtained all appropriate patient consent forms. In the form the patient(s) has/have given his/her/their consent for his/ her/their images and other clinical information to be reported in the journal. The patients understand that their names and initials will not be published and due efforts will be made to conceal their identity, but anonymity cannot be guaranteed.

\section{RÉFÉRENCES}

1. Camus F, Theunis A, de Saint-Aubain N, De Maubeuge J, Deraemaecker R, André J et al. Neurothécome du nez chez une adolescente. Ann Dermatol Venereol. 2012;139:165-7.

2. Fetsch JF, Laskin WB, Hallman JR, Lupton GP, Miettinen M. Neurothekeoma: an analysis of 178tumors with detailed immunohistochemical data and long-term patient follow-up information. Am J Surg Pathology. 2007;31:1103-14.

3. Brix M, Ranfaing E, Lesne F, Ricbourg B. Neurothécome cellulaire: un cas chez une enfant de 11 ans. Rev Stomatol Chir Maxillofac. 2005;106:356-9.

4. Barnhill RL, Mihm Jr MC. Cellular neurothekeoma. A distinctive variant of neurothekeoma mimicking nevomelanocytic tumors. Am J Surg Pathol. 1990;14:113-20.

5. Barnhill RL, Dickersin GR, Nickeleit V, Bhan AK, Muhlbauer JE, Phillips ME, et al. Studies on the cellular origin of neurothe- keoma. Clinical, light, microscopic, immunohistochemical and ultrastructural observations. JAAD. 1991;25:80-8.

Copyright by Fatima Zahra Elfatoiki, et al. This is an open-access article distributed under the terms of the Creative Commons Attribution License, which permits unrestricted use, distribution, and reproduction in any medium, provided the original author and source are credited.

Source of Support: Nil, Conflict of Interest: None declared. 